\title{
INTERPLANETARY SHOCK WAVES FROM MCMATH REGION 11976 DURING ITS PASSAGE IN AUGUST 1972
}

\author{
M. DR YER
}

Space Environment Laboratory, National Oceanic and Atmospheric Administration, Boulder, Colo. 80302, U.S.A.

A. EVIATAR, A. FROHLICH, A. JACOBS, and J. H. JOSEPH

Tel-Aviv University, Ramat-Aviv, Israel

and

E. J. WEBER

Kitt Peak National Observatory, Tucson, Ariz. 85717, U.S.A.

\begin{abstract}
J. Geophys. Res.). The August 1972 events provided an excellent opportunity for synthesizing a variety of observations in a coordinated fashion for the purpose of improving flare-shock associations, and our understanding of interplanetary shock propagation and solar wind interaction with planets and comets. These observations included the usual sudden commencements of magnetic storms at Earth; preliminary shock data from Heos-2, Prognoz-1 and Prognoz-2 (at Earth) and the radially-aligned Pioneers $9(0.77 \mathrm{AU})$ and $10(2.2 \mathrm{AU})$ located about $45^{\circ}$ east of the
\end{abstract}

\section{SPACECRAFT AND CELESTIAL OBJECTS IN ECLIPTIC PLANE \\ 2 - 11 AUGUST 1972 \\ (FIXED SUN - EARTH AXIS)}

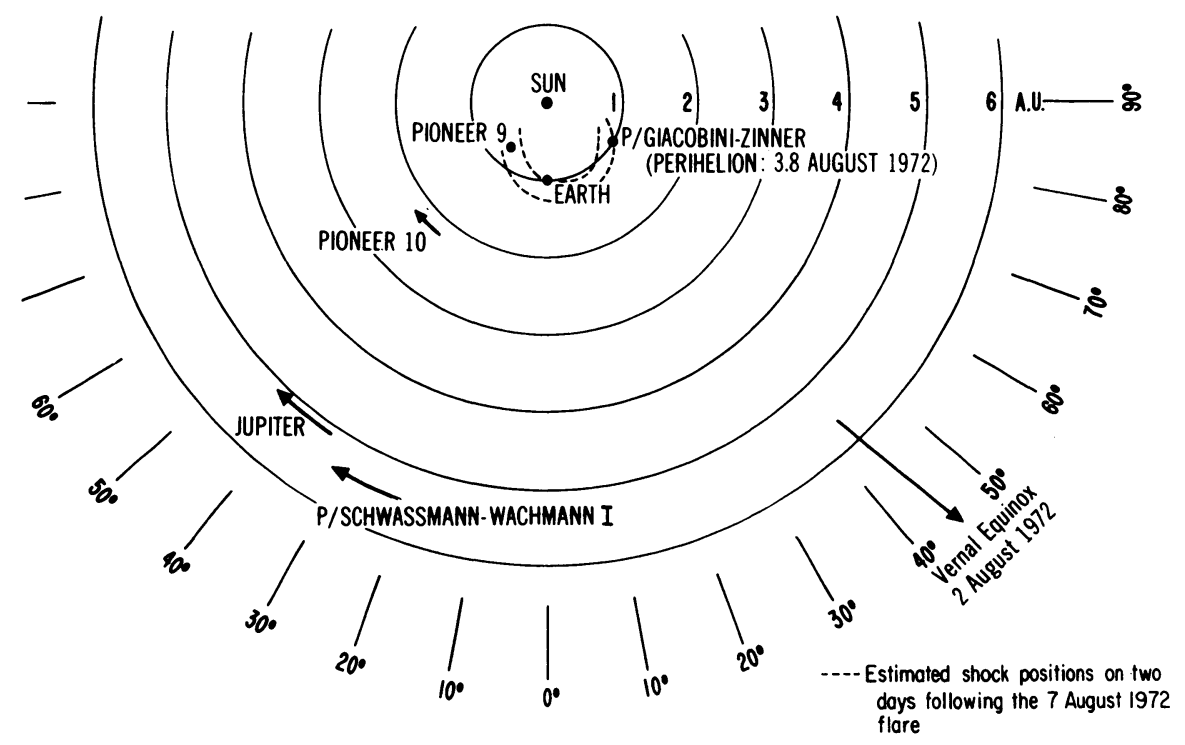

Fig. 1. Positions of Pioneers 9 and 10, Jupiter, and Comets $P /$ Giacobini-Zinner and $P /$ SchwassmannWachmann I relative to a fixed Sun-Earth axis during the epoch 1972 August 2-11. 
Sun-Earth axis; solar radio types II and IV (as reported in World Data Center A's UAG Report 28, 1973, and this Symposium); discrete radio source scintillations in the solar wind; and the more speculative ideas regarding the solar wind's interaction with planets and comets. In the last case, Jupiter and Comet $P /$ Schwassmann-Wachmann I exhibited non-Io-associated radio emission and a brightness increase, respectively, as a possible result of shock waves from the flare and/or coronal ejection activity initiated on 1972 June 15. During the August events, Comet $P /$ Giacobini-Zinner exhibited statistically-significant sudden brightness decrease following its perihelion on 1972 August 4 at $1 \mathrm{AU}$ approximately $57^{\circ}$ west of the Sun-Earth axis.

Figure 1 shows the various 'stations' in space. Shocks at Pioneers 9 and 10 were identified by detailed analysis of plasma and magnetic field data. SSCs at Earth and

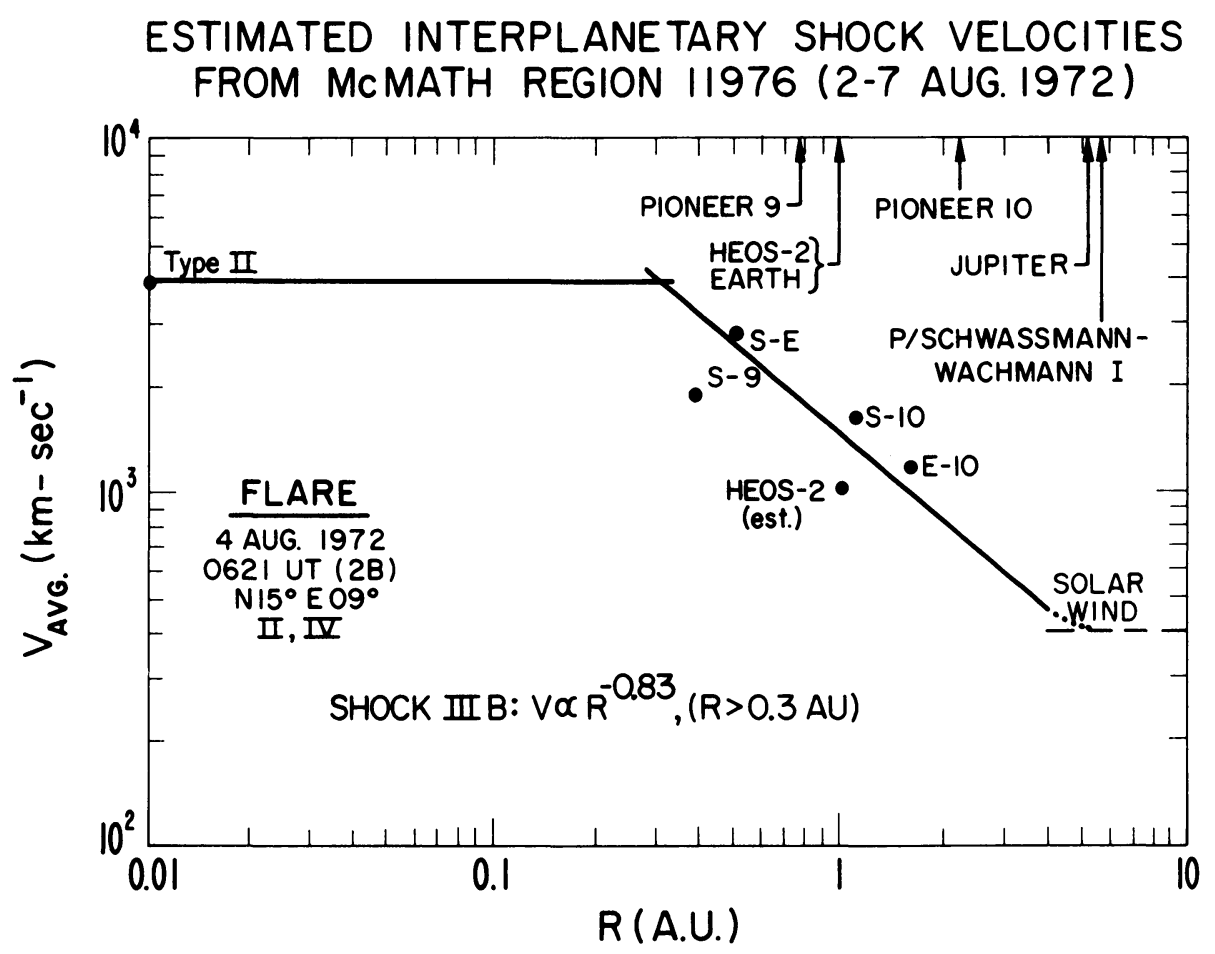

Fig. 2. Approximate shock trajectory from the flare on 1972 August 4 as inferred from the sudden commencement of a geomagnetic storm at Earth and in situ shock measurements observed at Pioneer 9 and Pioneer 10. The rapid deceleration suggests that (1) the energy input during the flare was impulsive thereby producing a 'blast' wave, or (2) the energy input produced a 'piston-driven' shock wave which decelerated rapidly after the cessation of the flare. The trajectory is 'approximate' in the sense that spherical symmetry is assumed. Thus, $V_{\mathrm{Avg}}=R_{i} /\left(t_{i}-t_{f}\right)$, where $R_{i}$ is the heliocentric radius to the measurement station, ' $i$ '; $t_{i}$ is the time of shock arrival at station, $i$; and $t_{f}$ is the time of the flare noted on the figure. Possible nonspherical collimation of the flare's shock wave and/or large-scale deformation of the shock by ambient solar wind inhomogenities are neglected. The various points, such as S-E, S-9, .. refer to the average shock velocities between the Sun and Earth, Sun and Pioneer 9, etc. In several cases, the points refer, for example, to the average velocities between Pioneer 9 and Pioneer 10. In this case, of course,

$$
V_{\mathrm{Avg}}=\Delta R /\left(t_{10}-t_{9}\right), \text { etc. }
$$


a brightness decrease of $P /$ Giacobini-Zinner on 1972 August 9 or 10 (followed by an equally precipitous intensity increase in the visible) were also used as timing devices. There was no response at either Jupiter or $P /$ Schwassmann-Wachmann I for reasons suggested on the basis of shock deceleration noted below.

The first interplanetary shock, denoted as Shock I, was ejected following the August 2 flare $\left(14^{\circ} \mathrm{N}, 35^{\circ} \mathrm{E}\right.$, optical $1 \mathrm{~B}$, types II and IV, $0316 \mathrm{UT}$ maximum). Its deceleration was fairly fast with a power law index of -1.1 for its variation of average velocity as a function of heliocentric distance. We have speculated that it was deviated from a collision course with Jupiter and $P /$ Schwassmann-Wachmann I by deceleration into an Alfvén wave.

Shock II originated in the flare at $1958 \mathrm{UT}$ on August $2\left(13^{\circ} \mathrm{N}, 28^{\circ} \mathrm{E}\right.$, optical $2 \mathrm{~B}$, types II and IV radio bursts). It, too, decelerated rapidly following a piston-driven effect from the Sun to $\sim 0.3 \mathrm{AU}$.

The third interplanetary shock (Shock III) was produced by the August 4 flare $\left(15^{\circ} \mathrm{N}, 09^{\circ} \mathrm{E}\right.$, optical 3B, types II and IV, $0621 \mathrm{UT}$ maximum). Its strong deceleration has a power index of -0.8 and is shown in Figure 2 . A high initial velocity is implied

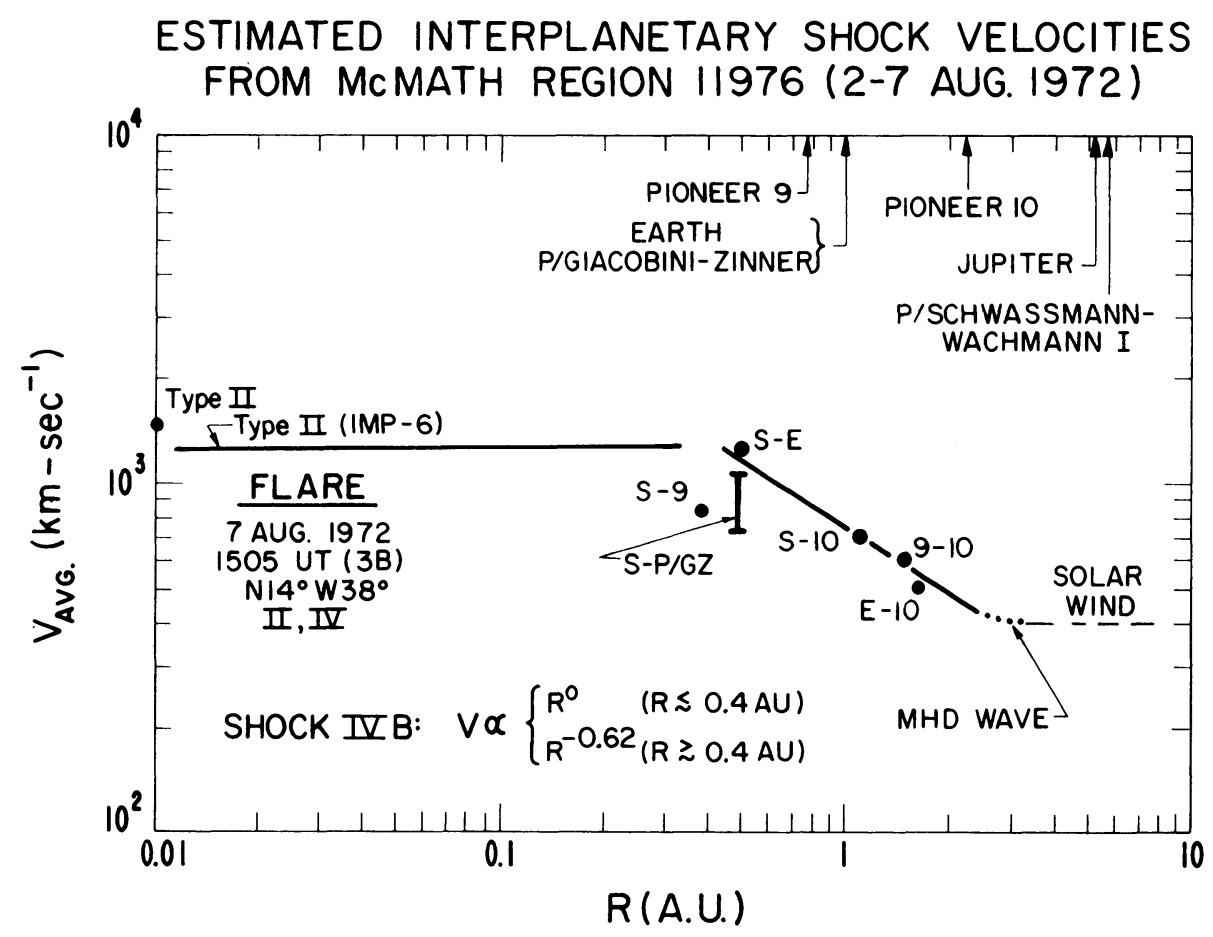

Fig. 3. Approximate shock trajectory from the flare on 1972 August 7 as inferred from the sudden commencement of a geomagnetic storm at Earth, low-frequency type II radio drifts observed by IMP 6, in situ shock measurements observed at Pioneers 9 and 10, and brightness diminution of Comet $P /$ GiacobiniZinner. The piston-driven character of the shock wave with fairly rapid deceleration beyond 0.5 to $1.0 \mathrm{AU}$ is likely for this case. Note that shock wave decay to an MHD wave is indicated prior to 5 AU if the ambient solar wind velocity is assumed to be $\sim 400 \mathrm{~km} \mathrm{~s}^{-1}$. 
at the Sun. More significantly, it appears to have decayed to a harmless Alfvén wave before reaching $5 \mathrm{AU}$ in a blast-wave fashion, following a similar piston motion.

Shock IV was produced by the August 7 flare $\left(14^{\circ} \mathrm{N}, 38^{\circ} \mathrm{W}\right.$, optical $3 \mathrm{~B}$, types II and IV, 1516 UT maximum) which probably produced a visual brightness decrease of $P /$ Giacobini-Zinner. We believe that this is possible when the usual solar wind flux of $\sim 10^{8} \mathrm{~cm}^{-2} \mathrm{~s}^{-1}$ is impulsively increased behind the shock (near the piston) to $\sim 10^{9} \mathrm{~cm}^{-2} \mathrm{~s}^{-1}$. Lifetime against impact dissociation for $\mathrm{C}_{2}$ (for example) is thereby decreased to about three days which is competitive with photodissociative processes. Figure 3 shows that the shock velocity was initially fairly constant (implying continuous energy outflow at the Sun) with a more blast-like deceleration thereafter. Again, this shock also appears to have decayed prior to reaching the $5 \mathrm{AU}$ position.

Detailed studies of these events will hopefully contribute to improved theories of interplanetary shock origin and propagation as well as solar wind interaction with cometary atmospheres. We do not wish to imply that the shocks noted above were the only ones produced by this spectacular display of flares. Additional shocks may indeed have been produced, and shock-shock interactions could have occurred in interplanetary space. Pending detailed analysis of all spacecraft data, including the various plasma and magnetic fluxes within the transient shock ensembles, we suggest that the shocks noted above are the essential discontinuities during the passage of McMath Region No. 11976 on the visible disk during 1972 August. Such analysis will also, hopefully, assist in the correlation of interplanetary events and the solar disturbances responsible for their existence.

\section{Acknowledgements}

We wish to express our sincere and deepest appreciation for comments, suggestions, and access to preliminary data from the following scientists: J. H. Wolfe, H. Collard, J. D. Mihalov, F. L. Scarf, D. S. Colburn, B. F. Smith, C. P. Sonett, A. Frosolone, J. McKinnon, G. Heckman, E. Roemer, G. McCorkle, J. W. Warwick, S. Zimny, H. H. Malitson, S. Cuperman, W. Bernstein, H. Rosenbauer, S. Pintér, J. Dodge and P. Hedgecock. A more complete report of this work has been submitted under the title 'Interplanetary Shock Waves and Comet Brightness Fluctuations during JuneAugust 1972', for possible publication in Journal of Geophysical Research.

\section{DISCUSSION}

Brandt: I would give the comet data very low weight in your study. F. D. Miller and B. Donn and J. Rahe have studied comet heads hit by interplanetary shocks and find no discernible effect on the comet. Hence, changes in cometary brightness or form may not be produced by shocks.

Dryer: We were looking for all possible effects of the shock. Scintillation data suggest that there were effects near the comet. The studies you cited were based upon a paucity of solar wind data.

Brandt: But comet workers searched for such effects and the evidence is negative.

Maxwell: I believe that $10^{4} \mathrm{~km} \mathrm{~s}^{-1}$ velocity deduced from the radio data by Dodge was based on a misidentification. We observed two components - one at $1000 \mathrm{~km} \mathrm{~s}^{-1}$ and one at $3000 \mathrm{~km} \mathrm{~s}^{-1}$.

Riddle (to Maxwell): The identification of the disturbance stated by Dodge to be a type II burst with a 
velocity of $9000 \mathrm{~km} \mathrm{~s}^{-1}$ is indeed in doubt. However, from the Boulder records there is no doubt that a disturbance with velocity $9000 \mathrm{~km} \mathrm{~s}^{-1}$ did exist, whatever its spectral type, as deduced by irregularities in the fringe lines on the interferometer record of a preexisting burst.

Sakurai: I would like to ask you what is the source of the piston which you say maintains the velocity before the deceleration? Is this related to the propagation of a moving type IV source in the solar atmosphere?

Dryer: I would like to give an affirmative answer, but no evidence exists between the observation at the sun and the still-sketchy plasma and field data observed by spacecraft. Regarding the latter, we have been speculating that anomalously-high alpha/proton abundance in the solar wind behind shocks $(\sim 0.15$, compared with the usual 0.04 ) is the tracer for the plasma piston or ejecta from the flare. We need additional information provided by theory to help us identify these pistons. The piston's velocity (as presently 'identified' as just noted) is about $80 \%$ of the shock velocity which preceded it by some $5 \mathrm{~h}$ or so. We would like to 'connect' these observations to the type IV moving source. As spacecraft get closer to the Sun we will get more information on this question. 